\title{
BMJ Open Internet-based cognitive behavioural therapy for adults with tinnitus in the UK: study protocol for a randomised controlled trial
}

\author{
Eldré W Beukes, ${ }^{1}$ Vinaya Manchaiah, ${ }^{2,3,4}$ Peter M Allen, ${ }^{1,5}$ David M Baguley, ${ }^{1,6}$ \\ Gerhard Andersson ${ }^{3,7}$
}

To cite: Beukes EW Manchaiah V, Allen PM, et al. Internet-based cognitive behavioural therapy for adults with tinnitus in the UK: study protocol for a randomised controlled trial. BMJ Open 2015;5:e008241. doi:10.1136/bmjopen-2015008241

- Prepublication history for this paper is available online. To view these files please visit the journal online (http://dx.doi.org/10.1136/ bmjopen-2015-008241).

Received 19 March 2015 Revised 19 July 2015 Accepted 18 August 2015

CrossMark

For numbered affiliations see end of article.

Correspondence to

Eldré W Beukes;

eldre.beukes@anglia.ac.uk

\section{ABSTRACT}

Introduction: Tinnitus is one of the most distressing hearing-related symptoms. Innovative ways of managing tinnitus distress and the related healthcare burden of treating tinnitus are required. An internetbased cognitive behavioural therapy (iCBT) intervention has been developed in Sweden to improve access to evidence-based tinnitus treatments. This study aims to determine the feasibility and effectiveness of iCBT in reducing the impact associated with tinnitus, in the UK. It, furthermore, aims to establish whether there are subgroups of tinnitus sufferers for whom this iCBT intervention may be more suitable.

Methods and analysis: A two-armed randomised control trial一with a 1-year follow-up design—will be used to evaluate the effectiveness of iCBT on tinnitus distress. A delayed treatment design using a weekly check-in control group will be used. 70 participants will be randomly assigned to each group by an independent researcher by using a computer-generated randomisation schedule, and after being prestratified for age and tinnitus severity. They will undergo the iCBT e-health intervention online together with audiological therapeutic support. The main outcome measure is the Tinnitus Functional Index. Process evaluation of the intervention will also be conducted. Data analysis will be in accordance with Consolidated Standards of Reporting Trials guidelines.

Ethics and dissemination: Ethical approval has been granted. If this intervention proves effective, it may be possible that at least some tinnitus sufferers can be managed though an iCBT e-learning treatment programme. This would be cost effective and potentially will free up services for those with more severe problems that need face-to-face treatment. Trial registration number: ClinicalTrials.gov; NCT02370810, date 05/03/2015.

\section{INTRODUCTION}

Tinnitus is one of the most distressing and debilitating hearing-related symptoms. ${ }^{1}$ Unwanted sounds, such as ringing or buzzing, are experienced in the absence of a

\section{Strengths and limitations of this study}

- An internet intervention may be a cost-effective way to manage the distress associated with tinnitus.

- Access to tinnitus treatment may improve as large numbers of adults can receive the intervention at one time.

- Considering results from the outcome measures together with the process evaluation findings will strengthen result interpretation.

- Being unable to blind the researcher and participants during the intervention may lead to study bias; however, the data analyst will be blinded to the groups.

- The participants recruited will be those who are motivated, able to set aside time for the intervention, and have the ability to use a computer; this mode of recruitment may not mimic the general tinnitus population.

concurrent external sound source. ${ }^{2}$ It is highly prevalent, affecting between $10 \%$ and $15 \%$ of adults in the UK, with an increased prevalence in older adults. ${ }^{3}$

Despite much research, medical treatments are usually ineffective at reducing tinnitus and a cure remains to be found. ${ }^{4}$ Tinnitus is, therefore, managed as a chronic condition, thus adding strain on current healthcare systems. ${ }^{5}$ For some, experiencing tinnitus can result in a complex set of symptoms. ${ }^{6}$ As there is a strong relationship between tinnitus and hearing difficulties, this adds to the distress experienced. ${ }^{7}$ In addition to the possible adverse effects on daily life, such as the impact on sleep, mood and concentration, there may also be a number of indirect psychological and psychosocial effects, including depression and anxiety. ${ }^{8}$ These concurrent physical and psychological effects add to the healthcare burden as further inputs may be required from various health professionals. ${ }^{9}$ 
When it comes to tinnitus management strategies, there is a low evidence-base for many practices. ${ }^{10}$ Good Practice Guidelines for tinnitus management were set by the Department of Health in the UK, in 2009. ${ }^{11}$ Hoare et $a l^{12}$ found poor guideline adherence, unequal access to care, lack of standardisation, and limited use of psychological interventions for tinnitus. Innovative ways of addressing these issues in the UK are, therefore, required.

Cognitive behavioural therapy (CBT) is a technique which reduces the distress associated with tinnitus, ${ }^{13}$ and is one of the tinnitus treatment options with the most evidence of effectiveness. ${ }^{14}$ It has been shown to add to the efficacy of audiology/ear, nose and throat (ENT) approaches to treatment. ${ }^{15}$ It is, however, rarely offered in clinical practice, ${ }^{16}$ particularly in the UK. ${ }^{17}$ This is largely due to a shortage of healthcare professionals with appropriate CBT training, such as psychologists who are welltrained Audiologists willing to manage tinnitus. ${ }^{18}$

To provide an accessible evidence-based tinnitus treatment, an internet-based CBT (iCBT) intervention was developed by Andersson et al, ${ }^{19}$ and results of their initial randomised control trial (RCT) showed promising effects. Following intervention improvements, further studies conducted in Sweden and Germany indicated similar results for face-to-face group CBT. ${ }^{20}{ }^{21} \mathrm{~A}$ further RCT in Australia, by Abbott et al, ${ }^{22}$ found results of iCBT did not show any statistically significant benefit over a information-only control programme (without CBT content). In addition, the attrition rate was higher in the iCBT group. This poses questions regarding whether the structure and presentation of the intervention requires updating to improve overall outcomes. If iCBT for tinnitus distress is feasible in the UK, it may bridge a gap for those who have not been able to access appropriate tinnitus services. It could, furthermore, reduce the burden on the healthcare system from those who do not require supra-specialist support.

A study to determine whether iCBT could be a suitable intervention is, therefore, of value. The aim of this study is to further our knowledge of the feasibility and effectiveness of iCBT for tinnitus management in the UK. This research is timely and in line with two of the tinnitus research priorities recommended by the James Lind Alliance, ${ }^{4}$ namely: research investigating which management strategies are more effective than the usual model of audiological care in improving outcomes for people with tinnitus, and research determining whether CBT, delivered by audiology professionals, is effective for people with tinnitus.

The research objectives for this study are, therefore, as follows:

1. To establish the feasibility of using iCBT for tinnitus distress as an intervention for adults with tinnitus in the UK;

2. To evaluate the effectiveness of iCBT for tinnitus distress in reducing the impact associated with tinnitus for adults in the UK;
3. To ascertain predictors of outcome for whom this iCBT intervention is a suitable intervention;

4. To determine the longer term effects of iCBT, 12-months postintervention.

\section{METHODS}

\section{Study design}

This will be an internet-based study run in the UK. This intervention study will be implemented as a two-armed RCT, with a 1-year follow-up, to evaluate the effectiveness of iCBT for tinnitus distress. An effectiveness trial has been selected to increase the extent to which the study results can be generalised, and will be used to identify for which subgroups of tinnitus sufferers this is a suitable intervention. A delayed treatment design using a weekly check-in (WCI) control group will be used, as shown in figure 1. The objective is to test the superiority of the intervention, with the hypothesis that the treatment group will perform better than the WCI group. These groups will be followed prospectively for 1 year, to establish the long-term effectiveness of the intervention programme.

This study design is described using SPIRIT (Standard Protocol Items: Recommendations for Interventional Trials) checklist. ${ }^{23}{ }^{24}$ and has been registered with Clinical Trials.gov: NCT02370810, date 05/03/2015.

\section{Study groups}

There will be two study groups. The Test Group will receive the 8-week duration iCBT treatment following baseline measurements and allocation (after $\mathrm{T}_{0}$ ). The WCI Control Group will be monitored during the active treatment phase by means of the Tinnitus Handicap Inventory-Screening version (THI-S), ${ }^{25}$ but will have no other contact or involvement in the study during this period. The control group will undergo the same iCBT intervention once the treatment group finishes the programme (after $\mathrm{T}_{1}$ ).

\section{Inclusion criteria}

A clinical evaluation prior to partaking in the study is desirable to rule out the presence of a serious auditory pathology or associated psychological problems, which may be related to having tinnitus. ${ }^{26}$ If there are any concerns, a letter will be provided to the participants to give to their general practitioner for further tinnitus management.

Participant's eligibility for the study is as follows:

1. Aged 18 years and over, and living in the UK;

2. The ability to read and type in English;

3. No barriers to using a computer (eg, significant fine motor control or visual problems);

4. Internet and email access, and the ability to use these;

5. Commitment to completing the programme;

6. Completion of the online screening and outcome questionnaires; 

design (iCBT, internet-based cognitive behavioural therapy; THI-S, Tinnitus Handicap Inventory-Screening version).
Figure 1 Flow chart of the study
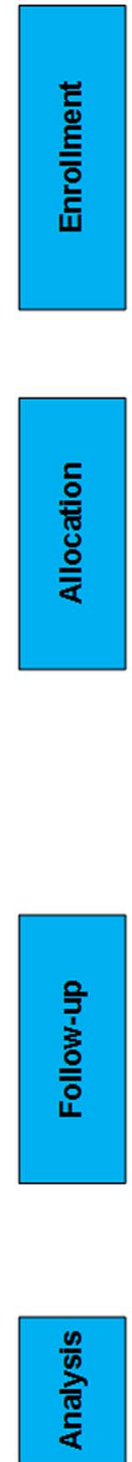

7. Agreeing to participate in either group and to be randomised to one of these groups;

8. Understanding and working towards the end goal of reducing the impact and distress of tinnitus, although the strength of the tinnitus may remain the same;

9. Be available for 12 months after starting the study to complete a 1-year follow-up questionnaire;

10. Suffering with tinnitus for a minimum period of 3 months;

11. Tinnitus outcome measure scores indicating the need for tinnitus care (26 or above on the Tinnitus Functional Index (TFI)). ${ }^{27}$

\section{Exclusion criteria}

1. Reporting any major medical or psychiatric conditions;

2. Reporting pulsatile, objective or unilateral tinnitus which have not been investigated medically;

3. Tinnitus is a consequence of a medical disorder and is still under investigation;



4. Undergoing any tinnitus therapy concurrently to the participation in this study.

A two-staged selection procedure will be followed:

1. An online screening questionnaire, which includes health and mental health-related questions;

2. A telephone interview during which the researcher will recheck eligibility, and provide the opportunity for potential participants to ask any questions related to the study.

\section{Recruitment}

Recruitment will be UK-wide, using as many different forms as possible to achieve adequate participant enrolment. Information about the study will be advertised at tinnitus and hearing-related support groups and charities, in their newsletters and drop-in centres. It will further be advertised in audiology departments and by using social media. Talks to professionals, such as ENT consultants and Audiologists, will also be given to introduce the study and aid recruitment. Those interested can obtain information and register interest on the study website. 


\section{Sample size}

Sample size estimation was calculated using Power and Sample Size Programme, V.3.1.2, and based on achieving a clinically relevant change of 13 points $(\mathrm{SD}=24.7)$ in the main outcome measure to be used for this study, the TFI. ${ }^{27}$ This indicated 30 participants were required per group-with an allocation ratio of 1:1-to achieve a twosided significance level of 0.05 and effect size of 0.8 . As these calculations are based on a fairly new outcome measure and there may be dropouts, an additional 40 participants will be added to ensure that there is enough valid data to draw conclusions from. Therefore, 70 participants will be assigned to the experimental group, and 70 participants to the control group. This is roughly in line with the sample sizes used in other similar studies.

\section{Allocation}

Participants will be randomly assigned to either the control or experimental group with a 1:1 allocation with the use of a computer-generated randomisation schedule (http://www.randomizer.org/) by an independent researcher. To prevent an unequal distribution among groups, participants will be prestratified on the basis of age $(\leq 60$ or $>60$ years) and tinnitus severity (TFI $\leq 50$ or $>50$ ). Furthermore, block randomisation, with blocks of four, will be applied to ensure equal group sizes within each criteria stratification.

Following allocation, participants will be contacted by telephone to provide to provide an opportunity for them to ask questions and discuss various aspects of the study. This initial contact has been found to be valuable to ensure participants are motivated to complete the treatment. ${ }^{20}$

\section{Intervention}

The intervention to be followed is built on an iCBT selfhelp programme for reducing tinnitus distress as developed by Andersson et al. ${ }^{19}$ It incorporates a combination of a cognitive rational ${ }^{28}$ and learning theory approach. ${ }^{29}$ The original intervention was largely text based. The present authors have redesigned and modified the intervention content and presentation to set up an interactive e-learning version, as shown in figure 2. This will ensure the intervention is visually stimulating, engaging, and responsive to participants' progress.

The intervention consists of fixed and optional modules, covered over a period of 8 weeks, as shown in table 1 . The modules contain a mixture of information, videos, quizzes, diagrams, suggested techniques to apply to daily life, worksheets to keep track of progress, solutions for common problems, and downloadable information.

The iCBT intervention will be delivered on a secure web platform, for which the participants will receive a password protected login information.

\section{Monitoring progress}

Participants will be minimally guided via a secure online messaging system. This therapeutic alliance will allow for feedback and assistance if participants have any difficulties, and has shown better outcomes than internet interventions without this communication. ${ }^{30}$ The clinician will contact the participants who have not completed their weekly progress worksheets to ensure that they are assisted as required. In these worksheets, participants record details about when, where, and for how long they practiced the suggested techniques, and how effective these techniques were.

As tinnitus therapy is largely delivered by the audiology community in the UK, an experienced audiological scientist, registered with the Health Professions Council, will undertake the role of supporting the participants; this will help to maintain consistency with the standard approach. Support will, however, be provided by a psychologist, with experience in iCBT interventions, and a protocol for dealing with different situations will be used.

\section{Assessment}

The assessment battery will consist of a screening eligibility questionnaire and relevant self-reported outcome measures that are related to areas which may be affected by tinnitus. Outcome measures for tinnitus severity, hearing handicap, insomnia, cognitive functioning, hyperacusis, anxiety, depression and life satisfaction were, therefore, selected. Careful consideration was given to ensure each questionnaire was as brief as possible and had good psychometric properties, as seen in table 2 . Also, the outcomes that were selected had to be aligned with the International Classification of Functioning, Disability and Health (ICF) ${ }^{31}$ framework in the domains of activity limitation and participation restriction.

The assessment battery and outcome measures will include:

1. Eligibility screening: A range of demographic, tinnitusspecific and health-related questions will be used to determine demographic variability and study eligibility. Open-ended questions asking about positive and negative experiences related to having tinnitus will also be included in the questionnaire.

2. The main outcome measures: The TFI has been selected to determine tinnitus severity, as it is validated for assessing responsiveness to treatment-both for scaling the severity and negative impact of tinnitus, and for measuring treatment-related changes in tinnitus. $^{27}$ The TFI was specifically designed to measure the effectiveness of interventions, a feature lacking in previous tinnitus outcome measures. ${ }^{32}$ Owing to its validation for assessing treatment responsiveness, it was given priority over some of the other more established questionnaires, such as the THI. ${ }^{33}$

3. Secondary outcome measures:

A. The THI-S ${ }^{25}$ will be used as a concise outcome measure, particularly to monitor tinnitus handicap on a weekly basis during the active treatment phase.

B. As there is a strong relationship between tinnitus and hearing difficulties, ${ }^{7}$ the Hearing Handicap 
Fixed modules

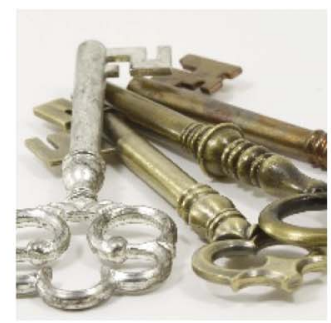

1. About this treatment

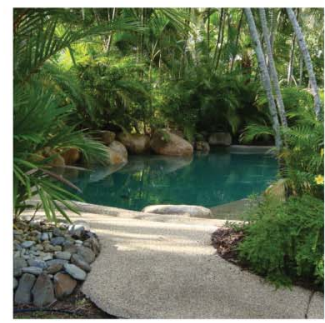

3. Relaxation: Step 1

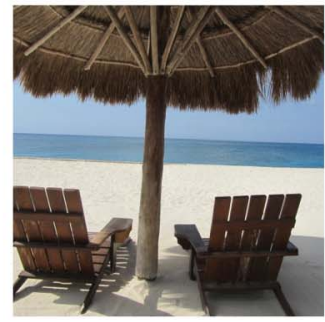

5. Relaxation: Step 2

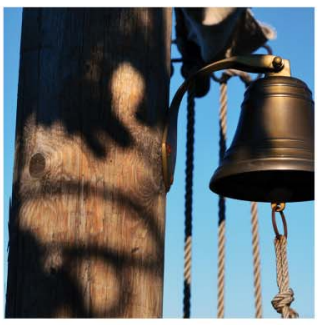

2. Tinnitus Overview

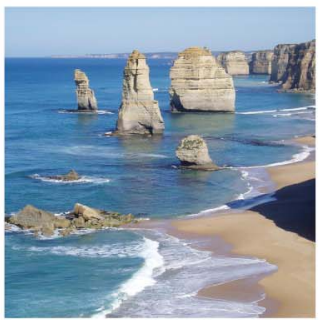

4. Positive imagery

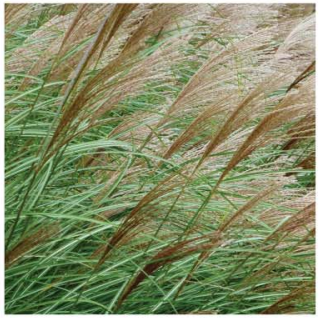

6. Reinterpretation of tinnitus
Optional modules

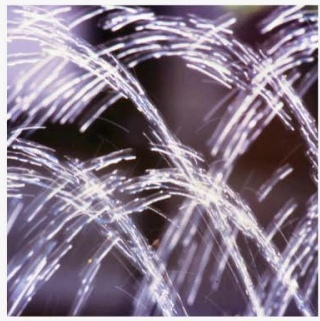

1. Sound enrichment

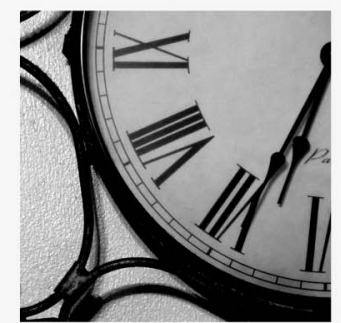

2. Sleep guidelines

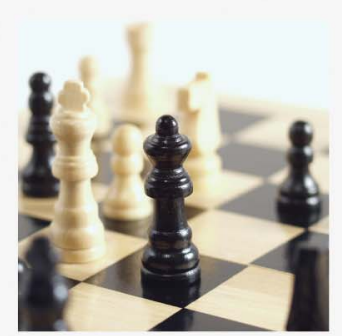

3. Concentration tips
Inventory for Adults-Screening version (HHIA-S $)^{34}$ will be administered to quantify perceived hearing handicap. It includes emotional and social/situational subscales.

C. As sleep disturbances are commonly associated with tinnitus, ${ }^{35}$ the Insomnia Severity Index $(\text { ISI })^{36}$ will be included. This questionnaire assesses the nature, severity, and impact of insomnia by assessing sleep duration, sleep quality, the negative impact on daily functioning, and psychological well-being.

D. As having tinnitus may disrupt cognitive functioning, ${ }^{37}$ the Cognitive Failures Questionnaire (CFQ) ${ }^{38}$ will be included, which has been designed to assess a patient's proneness to committing cognitive slips and errors in the completion of everyday tasks, such as failures in perception, memory, and motor functions.
E. As there is a high comorbidity of hyperacusis (a reduced tolerance for everyday sounds) ${ }^{39}$ in those with tinnitus, ${ }^{40}$ the Hyperacusis Questionnaire $^{41}$ will be administered. Although further work on the structure of this questionnaire is required to establish its reliability for measuring hyperacusis in the tinnitus research population, the scores obtained will be useful to compare preintervention and postintervention changes.

F. As anxiety and depression are common comorbid conditions in individuals with tinnitus, ${ }^{6}$ the Patient Health Questionnaire (PHQ-9), ${ }^{42}$ to measure depression severity, and the Generalised Anxiety Disorder 7-item (GAD-7) scale, ${ }^{43}$ to assess anxiety severity, will be administered.

G. To include a measure of quality of life, the Satisfaction with Life Scales ${ }^{44}$ will be used for assessing global life satisfaction. For the purpose 
Table 1 The components of the internet-based cognitive behavioural therapy (iCBT) intervention

\begin{tabular}{|c|c|c|c|}
\hline Week & Module & Explanation & Application \\
\hline \multirow[t]{2}{*}{1} & About this treatment & Introduction to the modules & Reading \\
\hline & Tinnitus overview & In-depth information & Quizzes \\
\hline \multirow[t]{3}{*}{2} & Relaxation: step 1 & Deep relaxation & 10-15 min, twice/day \\
\hline & $\begin{array}{l}\text { Identifying negative } \\
\text { thoughts }\end{array}$ & The link between thoughts and feelings & Writing down thoughts \\
\hline & Sound enrichment* & Using background sounds & Applying external sounds \\
\hline \multirow[t]{3}{*}{3} & Relaxation: step 2 & Diaphragmatic breathing & 5-7 min, twice/day \\
\hline & Cognitive restructuring & Analysing thoughts & Writing down situation, thoughts, feelings \\
\hline & Sleep guidelines* & Various techniques & Choose and apply techniques \\
\hline \multirow[t]{3}{*}{4} & Relaxation: step 3 & Entire body relaxation & 2-3 min, twice/day \\
\hline & Positive imagery & Use to enhance relaxation & Twice/day after relaxation \\
\hline & Concentration tips ${ }^{*}$ & Techniques discussed & Engage in mentally engaging activities \\
\hline \multirow[t]{3}{*}{5} & Relaxation: step 4 & Rapid relaxation & $20-60$ s, 5-10 times/day \\
\hline & Focus exercises & Mindful awareness & Twice/day after relaxation \\
\hline & Sensitivity to sound* & Gradual exposure & Listen to non-damaging, non-annoying sounds \\
\hline \multirow[t]{3}{*}{6} & Relaxation: step 5 & $\begin{array}{l}\text { Rapid relaxation in more difficult } \\
\text { situations }\end{array}$ & $30-60$ s, $10-15$ times/day \\
\hline & $\begin{array}{l}\text { Reinterpretation of } \\
\text { tinnitus }\end{array}$ & Change negative tinnitus associations & Writing about tinnitus thoughts \\
\hline & Hearing tactics* & Communication advice & Follow advice \\
\hline \multirow[t]{2}{*}{7} & Relaxation: step 6 & $\begin{array}{l}\text { Making relaxation part of daily routines } \\
\text { and habits }\end{array}$ & Rapid relaxation, 10-20 times/day \\
\hline & Exposure to tinnitus & $\begin{array}{l}\text { Decrease negative emotions and } \\
\text { avoidance of tinnitus }\end{array}$ & $\begin{array}{l}\text { Actively listen to tinnitus for } 5-10 \mathrm{~min} \text {, once/ } \\
\text { day, after relaxation }\end{array}$ \\
\hline \multirow[t]{2}{*}{8} & Key points summary & Highlighting key concepts & Online quiz \\
\hline & Future planning & Maintenance and relapse prevention & Making a plan to use tools in daily life \\
\hline
\end{tabular}

of this study, it was felt that the questions in this measure were more appropriate than those relating to longer quality of life measures, which include areas of mobility and self-care that are not directly targeted by this study.

Permission has been obtained to use the questionnaires whenever these are required, for example, the TFI.

\section{Questionnaire delivery}

The format of the questionnaire delivery will remain consistent in the study's online questionnaires. Not all questionnaires to be used have been validated for internet use. Previous research has found comparable results in terms of psychometric properties between computer and paper questionnaire delivery, with high test-retest reliability and completion rate on the internet. ${ }^{46}{ }^{47}$ See table 3 for the schedule of outcome measure delivery.

\section{Process evaluation}

An intervention, such as iCBT, is known as a 'complex intervention' as it combines different components, but does not involve drugs or surgical procedures. ${ }^{48}$ To fully investigate the different aspects of this complex intervention, we will be exploring the processes involved in implementing the iCBT intervention, as shown in figure 3, parallel to the iCBT study. By integrating this process

Table 2 Known psychometric properties of the questionnaires to be administered at some point during the study

\begin{tabular}{|c|c|c|c|}
\hline Measure and validation reference & Items & Scale used & $\begin{array}{l}\text { Internal consistency } \\
\text { (Cronbach's } \alpha)\end{array}$ \\
\hline Tinnitus Functional Index ${ }^{27}$ & 25 & $1-10$ & 0.97 \\
\hline Tinnitus Handicap Inventory-Screening ${ }^{25}$ & 10 & $1-3$ & 0.87 \\
\hline Hearing Handicap Inventory-Screening ${ }^{34}$ & 10 & $1-3$ & 0.93 \\
\hline Insomnia Severity Index ${ }^{36}$ & 7 & $0-4$ & 0.74 \\
\hline Cognitive Failures Questionnaire ${ }^{38}$ & 25 & $0-4$ & 0.89 \\
\hline Hyperacusis Questionnaire ${ }^{41}$ & 14 & $0-4$ & $0.66-0.68$ \\
\hline Patient Health Questionnaire ${ }^{42}$ & 9 & $0-3$ & 0.83 \\
\hline Generalised Anxiety Disorder ${ }^{45}$ & 7 & $0-3$ & 0.89 \\
\hline Satisfaction with Life Scales ${ }^{44}$ & 5 & $1-7$ & 0.87 \\
\hline
\end{tabular}


evaluation information with the outcome data, we will maximise our ability to interpret the results and the effectiveness of the intervention. ${ }^{49}$

We will be combining components from three evaluation models-the Reach, Efficacy, Adoption, Implementation and Maintenance (RE-AIM model) ${ }^{50} 51$ and the model designed by Linnan and Steckler, ${ }^{52}$ as well as that designed by Baranowski and Stables. ${ }^{53}$ The following eight components have been selected:

1. Recruitment: Procedures used to approach and attract participants.

2. Reach: The extent to which the targeted population were drawn to the study and were willing to be involved in this intervention study.

3. Context: Characteristics of the participants that affect the iCBT intervention, including social, demographical, socioeconomic factors.

4. Dose delivered: The number of modules and component included in this iCBT intervention.

5. Dose received: The extent to which participants actively engage and interact with the resources provided by this iCBT intervention.

6. Effectiveness: Examining the positive and negative consequences of the intervention, as well as factors that may positively or negatively influence the effectiveness of the intervention from the participant's perspective.

7. Maintenance: Keeping participants involved in this iCBT intervention and data collection.

8. Fidelity: The extent to which the intervention was implemented as planned.

These processes will be monitored during the intervention by reflecting on the study procedures and participants' experiences. After undergoing the intervention, participant satisfaction will be established using a Likert scale and telephone interview.

\section{Pilot study}

All materials will be piloted prior to running the study. Both professionals and those with tinnitus will test all aspects of the platform. A Likert scale questionnaire will be administered, asking specific questions related to the suitability, usability, content and experiences with the intervention and questionnaires, together with open-ended questions. The aim will be to identify any hindrances regarding use of the platform and possible barriers to participation. If any major changes are required, the ethical committee and trial body will be notified.

Further feasibility measures will include the recruitment rates, retention of participants, compliance and acceptability of the intervention by participants. The feasibility of having a professionally trained Audiologist running the intervention under supervision instead of a psychologist will also be evaluated.

\section{Data collection}

All data will be collected online. Baseline data will be collected at pretreatment $\left(-\mathrm{T}_{1}\right)$, prior to allocation.
During the active phase of the intervention, the responses to the 10 questions on the THI-S ${ }^{25}$ will be collected on a weekly basis. Data will then be collected at post-treatment $\left(\mathrm{T}_{1}\right)$ and after the control group completes the intervention $\left(\mathrm{T}_{2}\right)$. To determine long-term effectiveness of the intervention, data will again be collected 12 months $\left(\mathrm{T}_{\mathrm{x}}\right)$ after the start of the intervention at the close out, and this will end the study. The specific outcome measures for each collection point are shown in table 3 .

To improve rates at follow-up, an email will be sent to encourage participants to complete the questionnaires, with a maximum of three reminders. If they do not wish to further participate in the study, the reasons for their withdrawal will be recorded, where provided, by means of a post-treatment satisfaction questionnaire.

\section{Data management}

All participants will receive a non-traceable unique reference code to keep their identities blinded during the result analysis. All files used will be password protected. The researchers, statisticians and internal data monitoring committee (DMC) will have access to the final data set. The DMC includes researchers who are independent to this study, without competing interests. They will ensure accurate analysis and result interpretation.

\section{Data analysis}

Data analysis will be in accordance with Consolidated Standards of Reporting Trials (CONSORT) guidelines for randomised clinical trials. ${ }^{54}$ The SPSS V.20.0 will be used, and the data analyst will be blinded to the groups to minimise bias. Results at post-treatment will be based on an intention-to-treat paradigm, in which incomplete data sets will be analysed using multiple imputation offered by SPSS. For all analyses, a two-tailed significance level of $<0.05$ will be considered to be statistically significant. The data will be analysed, using a general linear model repeated measures approach, to look at the effects of the intervention and changes over time.

Qualitative content analysis with a positivist philosophical approach will be used to analyse the responses for open-ended questions. ${ }^{55}$ Integrating different analysis methods will yield further insight into the study outcomes. ${ }^{56}$

The study results will be shared in peer-reviewed publications by the present authors and presented at research conferences. A summary of the findings will be available to study participants, members of tinnitus support and tinnitus charity groups, as well as on Clinicaltrials.gov.

\section{Ethical considerations}

Participation is voluntary and all participants will provide informed consent online. A full explanation of every step of the study will be provided and participants will be able to withdraw at any stage without penalty.

A protocol has been established to ensure the security of participant's confidentiality on the web portal, 
Table 3 Schedule of enrolment, interventions and assessment

\begin{tabular}{|c|c|c|c|c|c|}
\hline \multicolumn{6}{|l|}{ Study period } \\
\hline \multirow[b]{2}{*}{ Time point } & \multirow{2}{*}{$\begin{array}{l}\text { Enrolment } \\
-T_{1}\end{array}$} & \multirow{2}{*}{$\begin{array}{l}\text { Allocation } \\
T_{0} \\
\end{array}$} & \multicolumn{2}{|l|}{ Postallocation } & \multirow{2}{*}{$\begin{array}{l}\text { Close out } \\
T_{x}(1 \text { year })\end{array}$} \\
\hline & & & $T_{1}(8$ weeks) & $T_{2}$ (16 weeks) & \\
\hline \multicolumn{6}{|l|}{ Enrolment } \\
\hline Eligibility screen & $\mathrm{x}$ & & & & \\
\hline Informed consent & $\mathrm{x}$ & & & & \\
\hline Allocation & & $\mathrm{x}$ & & & \\
\hline \multicolumn{6}{|l|}{ Interventions } \\
\hline Experimental group & & $\leqslant$ & $>$ & & \\
\hline Control group & & & $\leftarrow$ & $\approx$ & \\
\hline \multicolumn{6}{|l|}{ Assessments } \\
\hline Tinnitus Functional Index & $\mathrm{x}$ & & $\mathrm{x}$ & $\mathrm{x}$ & $\mathrm{x}$ \\
\hline Tinnitus Handicap Inventory_Screening version & $\mathrm{x}$ & X (weekly) & $\mathrm{x}$ & $\mathrm{x}$ & $\mathrm{x}$ \\
\hline Hearing Handicap Inventory-Screening version & $\mathrm{x}$ & & $\mathrm{x}$ & $\mathrm{x}$ & $\mathrm{x}$ \\
\hline Insomnia Severity Index & $\mathrm{x}$ & & $\mathrm{x}$ & $\mathrm{x}$ & $\mathrm{x}$ \\
\hline Cognitive Failures Questionnaire & $\mathrm{x}$ & & $\mathrm{x}$ & $\mathrm{x}$ & $\mathrm{x}$ \\
\hline Hyperacusis Questionnaire & $\mathrm{x}$ & & $\mathrm{x}$ & $\mathrm{x}$ & $\mathrm{x}$ \\
\hline Patient Health Questionnaire & $\mathrm{x}$ & & $\mathrm{x}$ & $\mathrm{x}$ & $\mathrm{x}$ \\
\hline Generalised Anxiety Disorder & $\mathrm{x}$ & & $\mathrm{x}$ & $\mathrm{x}$ & $\mathrm{x}$ \\
\hline Satisfaction with Life & $\mathrm{x}$ & & $\mathrm{x}$ & $\mathrm{x}$ & $\mathrm{x}$ \\
\hline
\end{tabular}

complying with European guidelines for internet studies. Participants' data will be anonymised as unique reference codes will be used. Protocols to minimise the risks to participants and the researcher have been put in place. The data together with any other spontaneously reported adverse events during the intervention will be reported. If any participants were identified as requiring additional support, a letter will be provided to them to show to their general practitioner so that this care can be arranged.

\section{DISCUSSION}

In this paper, the design of a study to investigate the effectiveness and feasibility of an internet-based intervention for tinnitus in the UK is outlined. The strength of the proposed methodology is the randomised design. A further strength is that not only the effects of the iCBT intervention, but also those from the process of evaluation, will be investigated, so as to fully determine the intervention's feasibility and effectiveness.
Figure 3 The processes to be evaluated following the study.

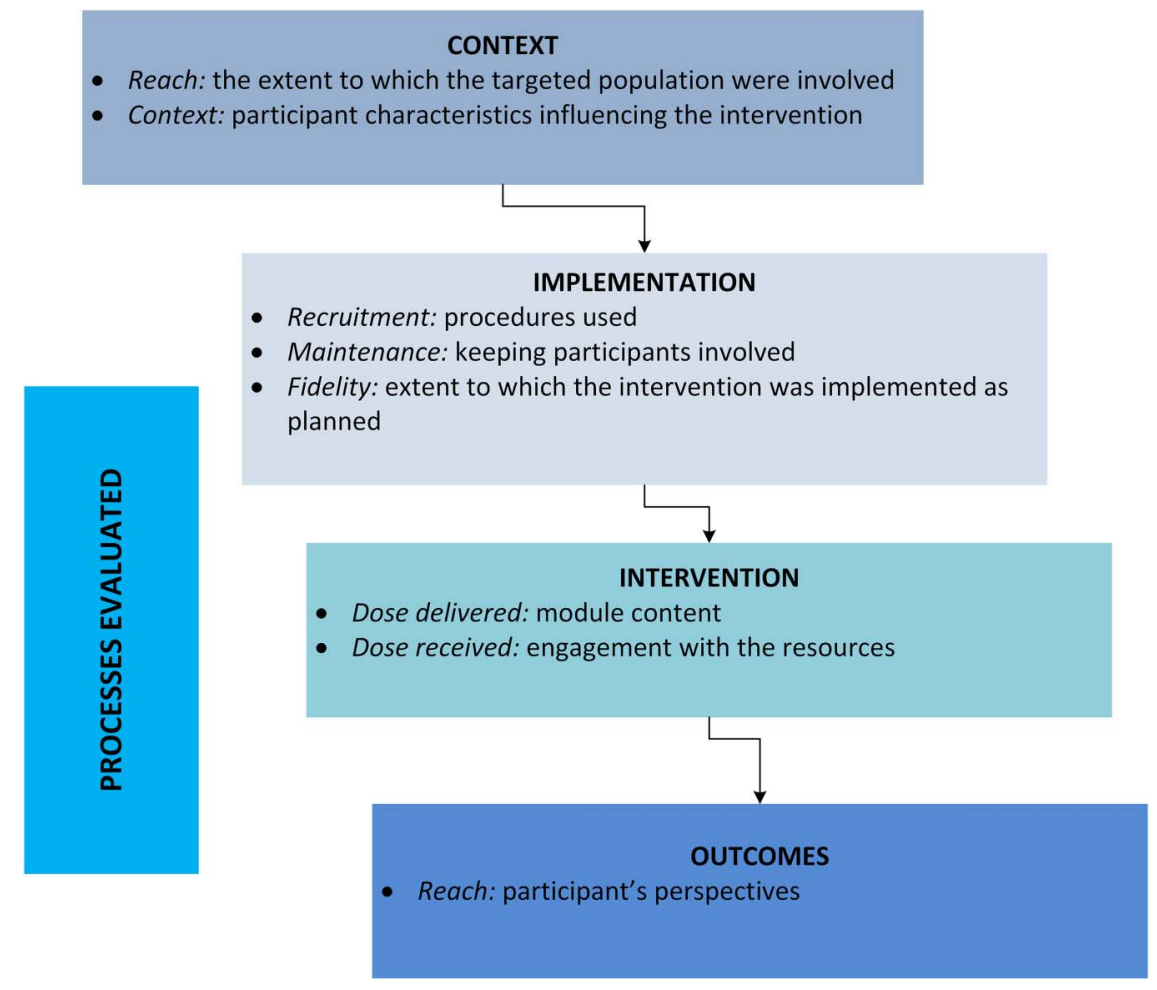


This particular tinnitus intervention has been selected due to the numerous potential benefits it may have. It is a standardised treatment, in which each participant has access to the same materials. The researchers have, however, carefully considered how to improve on methods used in similar previous studies and built on these. The following improvements will be made during this study. First, redesigning the module content and combining both CBT and audiology principles, thereby ensuring it is multidisciplinary in nature. Second, transforming the presentation of materials into an interactive e-learning version, which is visually stimulating and may help improve participant engagement. Third, using a main outcome measure that is specifically designed for measuring intervention effects. Fourth, assessing a range of outcomes to help identify for whom this intervention is most suited. Fifth, enabling the intervention to be presented by an Audiologist instead of a Psychologist. Finally, aiming for good participant retention by use of careful inclusion criteria and screening methods.

There are, however, potential barriers that can be forecasted for this study. Although according to the 2015 report from the Office of Statistics, ${ }^{57} 86 \%$ of the population in Great Britain have internet access, there is still a proportion who do not have access and will, therefore, not be able to participate in this study. Those without access may be older adults, which might impact the sample selected. There are also people with visual or motor disabilities who will be unable to use a computer effectively and who, therefore, may not be suitable for this intervention unless they get assistance. A further barrier is that participants will require motivation to complete the questionnaires and treatment modules. A limitation of the study design is that it is not possible to blind the researcher and participants during the intervention as they will know in which group they have been placed. Bias will, however, be minimised by stratification and blinding during randomisation and data analysis.

Feasibility and uptake of such an intervention will be determined by this study. It is, however, encouraging that other internet-based studies run within the UK, for other health-related concerns, ${ }^{58}{ }^{59}$ have reported sufficient interest. Owing to the multidisciplinary nature of the study, it furthermore has the prospect of determining the effects of the iCBT intervention on tinnitus outcomes, as well as on the person globally as various comorbid factors are being investigated.

The potential impact of this research is that it can change the way in which tinnitus services are delivered in the UK. If effective, iCBT may be suggested for certain tinnitus sufferers following their clinical examination. This will address differential clinical demands and reduce the number of tinnitus sufferers needing face-to-face consultations. This may in turn diminish the current burden on the National Health Service healthcare and lead to a significant potential cost saving to the health service. It may also make treatment available to many who are unable to access speciality tinnitus services.
If this intervention is feasible, future studies should focus on comparing iCBT to the usual tinnitus care in the UK. It is expected that the results of this study will become available early in 2017 .

\section{Author affiliations}

${ }^{1}$ Department of Vision and Hearing Sciences, Anglia Ruskin University, Cambridge, UK

${ }^{2}$ Department of Speech and Hearing Sciences, Lamar University, Beaumont, Texas, USA

${ }^{3}$ Department of Behavioral Sciences and Learning, Linköping University, Linköping, Sweden

${ }^{4}$ Audiology India, Mysore, Karnataka, India

${ }^{5}$ Vision and Eye Research Unit, Anglia Ruskin University, Cambridge, UK

${ }^{6}$ Audiology Department, Cambridge University Hospital NHS Foundation Trust, Cambridge, UK

${ }^{7}$ Department of Clinical Neuroscience, Division of Psychiatry, Karolinska Institute, Stockholm, Sweden

Acknowledgements The authors are grateful to Linköping University for hosting the web portal for this study and to their webmaster, George Vlaescu, for the technical assistance.

Contributors GA, VM and DMB conceived this study. EWB designed the study protocol with substantial contributions from GA, VM, PMA and DMB.

The intervention was developed by GA, and subsequently modified by EWB. The manuscript was drafted by EWB, who is also responsible for the overall operational aspects of this study. All the authors critically revised and approved the manuscript.

Competing interests None declared.

Ethics approval Ethical approval has been granted by the Research Ethics Committee (REC) of Anglia Ruskin University (FST/FREP/14/478).

Provenance and peer review Not commissioned; externally peer reviewed.

Data sharing statement All the data used in the project will be generated directly as a result of the project, without any pre-existing data being used. All data generated during the project will be made available. DOls to these data will be provided and cited in any published articles using this data and any other data generated.

Open Access This is an Open Access article distributed in accordance with the Creative Commons Attribution Non Commercial (CC BY-NC 4.0) license, which permits others to distribute, remix, adapt, build upon this work noncommercially, and license their derivative works on different terms, provided the original work is properly cited and the use is non-commercial. See: http:// creativecommons.org/licenses/by-nc/4.0/

\section{REFERENCES}

1. Cima RFF, Vlaeyen JWS, Maes IHL, et al. Tinnitus interferes with daily life activities: a psychometric examination of the Tinnitus Disability Index. Ear Hear 2011;32:623-33.

2. McFadden D. Tinnitus: facts, theories, and treatments. National Academies, 1982.

3. Davis A, Rafaie EA. Epidemiology of tinnitus. In: Tyler R, ed. Tinnitus handbook. San Diego: Singular, 2000:1-23.

4. Hall DA, Mohamad N, Firkins L, et al. Identifying and prioritizing unmet research questions for people with tinnitus: the James Lind Alliance Tinnitus Priority Setting Partnership. Clin Invest 2013;3:21-8.

5. Maes IH, Cima RF, Vlaeyen JW, et al. Tinnitus: a cost study. Ear Hear 2013;34:508-14.

6. Belli S, Belli H, Bahcebasi T, et al. Assessment of psychopathological aspects and psychiatric comorbidities in patients affected by tinnitus. Eur Arch Otorhinolaryngol 2008;265:279-85.

7. Palmer KT, Griffin MJ, Syddall HE, et al. Occupational exposure to noise and the attributable burden of hearing difficulties in Great Britain. Occup Environ Med 2002;59:634-9.

8. Bartels $\mathrm{H}$, Middel BL, van der Laan BF, et al. The additive effect of co-occurring anxiety and depression on health status, quality of life 
and coping strategies in help-seeking tinnitus sufferers. Ear Hear 2008;29:947-56.

9. Cima RMJ, Joore M, Maes I, et al. Cost-effectiveness of multidisciplinary management of Tinnitus at a specialized Tinnitus centre. BMC Health Serv Res 2009;9:29.

10. Hoare DJ, Kowalkowski VL, Kang S, et al. Systematic review and meta-analyses of randomized controlled trials examining tinnitus management. Laryngoscope 2011;121:1555-64.

11. Department of Health. Provision of services for adults with tinnitus. A Good Practice Guide. London, England: Central Office of Information, 2009

12. Hoare DJ, Gander PE, Collins L, et al. Management of tinnitus in English NHS Audiology Departments: an evaluation of current practice. J Eval Clin Pract 2012;18:326-34

13. Cima RF, Andersson G, Schmidt CJ, et al. Cognitive-behavioral treatments for tinnitus: a review of the literature. J Am Acad Audiol 2014;25:29-61.

14. Hesser $\mathrm{H}$, Weise $\mathrm{C}$, Westin VZ, et al. A systematic review and meta-analysis of randomized controlled trials of cognitive-behaviora therapy for tinnitus distress. Clin Psychol Rev 2011;31:545-53.

15. Cima RF, Maes IH, Joore MA, et al. Specialised treatment based on cognitive behaviour therapy versus usual care for tinnitus: a randomised controlled trial. Lancet 2012;379:1951-9.

16. Gander PE, Hoare DJ, Collins L, et al. Tinnitus referral pathways within the National Health Service in England: a survey of their perceived effectiveness among audiology staff. BMC Health Serv Res 2011;11:162

17. Baguley D, McFerran D, Hall D. Tinnitus. Lancet 2013;382:1600-7.

18. McFerran $D$, Baguley $D$. Is psychology really the best treatment for tinnitus? Clin Otolaryngoly 2009;34:99-101.

19. Andersson G, Strömgren T, Ström L, et al. Randomized controlled trial of internet-based cognitive behavior therapy for distress associated with tinnitus. Psychosom Med 2002;64:810-16.

20. Jasper K, Weise C, Conrad I, et al. Internet- based guided self-help versus group cognitive behavioral therapy for chronic tinnitus: a randomized controlled trial. Psychother Psychosom 2014;83:234-46.

21. Kaldo V, Levin S, Widarsson J, et al. Internet versus group cognitive-behavioral treatment of distress associated with tinnitus: a randomized controlled trial. Behav Ther 2008;39:348-59.

22. Abbott JM, Kaldo V, Klein B, et al. A cluster randomised trial of an internet-based intervention program for tinnitus distress in an industrial setting. Cogn Behav Ther 2009;38:162-73.

23. Chan A, Tetzlaff JM, Altman DG, et al. SPIRIT 2013 statement: defining standard protocol items for clinical trials. Ann Intern Med 2013;158:200-7.

24. Chan A, Tetzlaff JM, Gøtzsche PC, et al. SPIRIT 2013 explanation and elaboration: guidance for protocols of clinical trials. $B M J$ 2013;346:e7586.

25. Newman CW, Sandridge SA, Bolek L. Development and psychometric adequacy of the screening version of the Tinnitus Handicap Inventory. Otol Neurotol 2008;29:276-81.

26. Baguley D, Andersson G, McFerran D, et al. "Medical Models of Tinnitus." Tinnitus: a multidisciplinary approach. 2nd ed. John Wiley \& Sons, 2012:43-66

27. Meikle MB, Henry JA, Griest SE, et al. The Tinnitus Functional Index: development of a new clinical measure for chronic, intrusive tinnitus. Ear Hear 2012;33:153-76.

28. Henry JL, Wilson PH. The psychological management of chronic tinnitus: a cognitive-behavioral approach. Allyn \& Bacon, 2001.

29. Hallam R, Rachman S, Hinchcliffe R. Psychological aspects of tinnitus. Contrib Med Psychol 1984;3:31-53.

30. Andersson G, Carlbring P, Berger T, et al. What makes internet therapy work? Cogn Behav Ther 2009;38(S1):55-60.

31. World Health Organization. International Classification of Functioning, Disability And Health (ICF). Geneva, Switzerland: WHO, 2001

32. Kamalski DM, Hoekstra CE, van Zanten BG, et al. Measuring disease-specific health-related quality of life to evaluate treatment outcomes in tinnitus patients: a systematic review. Otolaryngol Head Neck Surg 2010;143:181-5.

33. Newman CW, Jacobson GP, Spitzer JB. Development of the Tinnitus Handicap Inventory. Arch Otolaryngol-Head Neck Surg 1996;122:143-8.
34. Newman CW, Weinstein BE, Jacobson GP, et al. Test-retest reliability of the Hearing Handicap Inventory for Adults. Ear Hear 1991;12:355-7.

35. Crönlein T, Langguth $B$, Geisler $P$, et al. Tinnitus and insomnia. Prog Brain Res 2007;166:227-33.

36. Bastien $\mathrm{CH}$, Vallières $\mathrm{A}$, Morin $\mathrm{CM}$. Validation of the Insomnia Severity Index as an outcome measure for insomnia research. Sleep Med 2001;2:297-307.

37. Andersson G, McKenna L. The role of cognition in tinnitus. Acta Otolaryngol 2006;126(S556):39-43.

38. Broadbent DE, Cooper PF, FitzGerald P, et al. The Cognitive Failures Questionnaire (CFQ) and its correlates. Br J Clin Psychol 1982;21(Pt 1):1-16.

39. Vernon JA. Pathophysiology of tinnitus: a special case-hyperacusis and a proposed treatment. Am J Otol 1987;8:201-2.

40. Sood S, Coles R. Hyperacusis and phonophobia in tinnitus patients. Br J Audiol 1998;22:228.

41. Khalfa S, Dubal S, Veuillet E, et al. Psychometric normalization of a hyperacusis questionnaire. ORL J Otorhinolaryngol Relat Spec 2002;64:436-42.

42. Spitzer RL, Kroenke K, Williams JB, Patient Health Questionnaire Primary Care Study Group. Validation and utility of a self-report version of PRIME-MD: the PHQ primary care study. JAMA 1999;282:1737-44.

43. Spitzer RL, Kroenke K, Williams JB, et al. A brief measure for assessing Generalized Anxiety Disorder: the GAD-7. Arch Intern Med 2006;166:1092-7.

44. Diener E, Emmons RA, Larsen RJ, et al. The Satisfaction with Life Scale. J Pers Assess 1985;49:71-5.

45. Lowe B, Decker O, Muller S, et al. Validation and standardization of the Generalized Anxiety Disorder Screener (GAD-7) in the general population. Med Care 2008;46:266-74.

46. Ritter $\mathrm{P}$, Lorig K, Laurent $\mathrm{D}$, et al. Internet versus mailed questionnaires: a randomized comparison. J Med Internet Res 2004;6:e29.

47. Thoren ES, Andersson G, Lunner T. The use of research questionnaires with hearing impaired adults: online vs. paper-andpencil administration. BMC Ear Nose Throat Disord 2012;12:12.

48. Campbell M, Fitzpatrick R, Haines A, et al. Framework for design and evaluation of complex interventions to improve health. BMJ 2000;321:694-6.

49. Moore G, Audrey S, Barker M, et al. Process evaluation of complex interventions. Medical Research Council guidance. BMJ 2015;350: h1258 350-1238

50. Glasgow RE, Vogt TM, Boles SM. Evaluating the public health impact of health promotion interventions: the RE-AIM framework. Am J Public Health 1999;89:1322-7.

51. Dzewaltowski DA, Glasgow RE, Klesges LM, et al. RE-AIM evidence-based standards and a Web resource to improve translation of research into practice. Ann Behav Med 2004;28: 75-80.

52. Linnan L, Steckler AB. Process evaluation for public health interventions and research. CA: Jossey-Bass San Francisco, 2002.

53. Baranowski T, Stables G. Process evaluations of the 5-a-day projects. Health Educ Behav 2000;27:157-66.

54. Schulz KF, Altman DG, Moher D. CONSORT 2010 Statement: updated guidelines for reporting parallel group randomised trials. BMC Med 2010:8:18.

55. Graneheim UH, Lundman B. Qualitative content analysis in nursing research: concepts, procedures and measures to achieve trustworthiness. Nurse Educ Today 2004;24:105-12.

56. Teddlie C. Foundations of mixed methods research: integrating quantitative and qualitative approaches in the social and behavioral sciences. Foundations of Mixed Methods Research, 2009.

57. Office for National Statistics. Internet access: households and individuals. London: Her Majesty's Stationary Office, 2015.

58. Brown J, Michie S, Geraghty AW, et al. Internet-based intervention for smoking cessation (StopAdvisor) in people with low and high socioeconomic status: a randomised controlled trial. Lancet Respir Med 2014;2:997-1006.

59. Moss-Morris R, McCrone P, Yardley L, et al. A pilot randomised controlled trial of an Internet-based cognitive behavioural therapy self-management programme (MS Invigor8) for multiple sclerosis fatigue. Behav Res Ther 2012;50:415-21. 